\title{
Vocal expression of emotional valence in pigs across multiple call types and contexts
}

\author{
Elodie F Briefer ${ }^{1}$, Pavel Linhart², Richard Policht ${ }^{2}$, Marek Špinka², Lisette Leliveld ${ }^{3}$, Sandra Düpjan ${ }^{3}$, \\ Birger Puppe ${ }^{3}$, Mónica Padilla de la Torre ${ }^{4}$, Andrew M. Janczak ${ }^{4}$, Cécile Bourguet ${ }^{5}$, Véronique Deiss ${ }^{6}$, \\ Alain Boissy ${ }^{6}$, Carole Guérin ${ }^{7}$, Eva Read ${ }^{7}$, Marjorie Coulon $^{8}$, Edna Hillmann ${ }^{9}$, Céline Tallet ${ }^{7}$ \\ ${ }^{1}$ Institute of Agricultural Sciences, ETH Zürich, Zürich, Switzerland \\ ${ }^{2}$ Institute of Animal Science, Prague, Czech Republic \\ ${ }^{3}$ Leibniz Institute for Farm Animal Biology, Germany \\ ${ }^{4}$ Department of Production Animal Clinical Sciences, Norwegian University of Life Sciences, Norway \\ ${ }^{5}$ Bureau E.T.R.E., France \\ 6 INRA UMR 1213 Herbivores, France \\ 7 INRA UMR 1348 PEGASE, France \\ ${ }^{8}$ Cabinet EASIER, France \\ ${ }^{9}$ Humboldt-Universität zu Berlin, Germany
}

Corresponding Author:

Elodie F Briefer ${ }^{1}$

Present address: University of Copenhagen, Copenhagen $\varnothing, 2100$, Denmark

Email address: elodie.briefer@bio.ku.dk

\section{Introduction}

Emotions, unlike mood, are short-lived reactions associated with specific events. They can be characterized by two main dimensions, their arousal (bodily activation) and valence (negative versus positive) (Mendl et al. 2010). Knowledge of the valence of emotions experienced by domestic and captive animals is crucial for assessing and improving their welfare, as it enables us to minimize the negative emotions that they might experience and to promote positive ones. Emotions can affect vocalizations directly or indirectly through the brain, lungs, larynx or vocal tract. As a result, vocal expression of emotions has been observed across species (Briefer 2012), and could serve as a non-invasive and potentially very reliable tool to assess animal emotions. In pigs (Sus scrofa), vocal expression of emotions has been relatively well studied (e.g. Leliveld et al. 2016; Briefer et al. 2019). However, it is not known if the vocal indicators revealed in previous studies are valid across call types and contexts. To find this out, we conducted a meta-analysis of the effects of emotional valence on pig vocalizations, including calls recorded in the most common emotional situations encountered by pigs throughout their lives, from birth to slaughter.

\section{Materials \& Methods}

\section{Recordings}

Pigs of various ages (piglets to finishing pigs) were recorded in 22 contexts triggering both negative emotions (e.g. crushing, missed nursing, castration, fear conditioning, isolation, restraint, barren environment, and slaughter), and positive emotions (e.g. nursing, huddling, social reunion, exposition to an enriched arena, and running) (for more details see Briefer et al. 2019; Illmann et al. 2013; Tallet et al. 2013; Linhart et al. 2015; Leliveld et al. 2016). The putative valence of the various contexts was based on the function of emotions to trigger avoidance (negative emotions) or approach (positive emotions) and the behavior of the pigs (Mendl et al. 2010).

\section{Vocal analyses}

In order to exclude very short sounds, in which parameters might not be accurately measured, only high quality calls with a duration $>0.05 \mathrm{~s}$ were selected for the acoustic analysis ( $n=6017$ calls). We used the 
acoustic features of the calls to classify them as low-frequency stable, modulated or tonal calls, highfrequency stable or modulated calls, or mixed calls (6 types), based on Tallet et al. (2013). Then, depending on the call type, we extracted 11 to 18 vocal parameters using a custom-built script in Praat, which batch-processed the analyses and the exporting of output data. The measured parameters belonged to the six following categories: source-related (fundamental frequency, "F0"), energy spectrum distribution, duration, amplitude modulation ("AM"), noise, filter-related (vocal tract resonances).

\section{Statistical analyses}

To eliminate redundancy, we used a principal component analysis to select one vocal parameter within each category, which explained most of the variance in the data across all call types, for further analyses. Since the minimum formant dispersion ("DFmin"), originally categorized along with the linear predictive coding ("LPC") coefficients never associated (i.e. loaded highly ( $r>=10.5 \mathrm{I}$ ) on the same PC) with these parameters, it was analyzed separately. These selected seven parameters (i.e. one for each of the six categories and DFmin; Table 1) were then used as outcome variables in linear mixed-effects models (Imer function in R software), to assess if they were affected by the valence of the contexts (positive or negative; fixed factor). The models included as control factors the age category and the call type. The context of production nested within the identity of the pig, nested within the experiment number, nested within the team who performed the recording was added as a random factor to control for repeated measurements and dependencies. The p-values were calculated with parametric bootstrap tests.

\section{Results and Discussion}

Five of the seven tested vocal parameters were affected by the valence of the context (Table 1). After controlling for the type of call and the age category (control factors), our analyses revealed that pigs produced calls characterized by a higher center of gravity, a shorter duration, less noise (lower Wiener entropy), lower formants (measured using the formant dispersion) and LPC coefficients in positive compared to negative contexts.

Table 1. Model estimates, lower (lo.ci) and upper (up.ci) 95\% confidence intervals for the vocal parameters included in the linear mixed-effect models, as a function of the valence of the contexts $\left({ }^{*} p<0.05 ;{ }^{* *} p<0.01 ;\right.$ "NS" Non significant).

\begin{tabular}{clrrrr}
\hline Parameter & Valence & estim & Io.ci & up.ci & P value \\
\hline Mean F0 (Hz) & Pos & 132.91 & 124.81 & 141.13 & NS \\
& Neg & 138.19 & 130.49 & 146.26 & \\
Spectral centre of gravity (Hz) & Pos & 967.70 & 877.57 & 1084.53 & $*$ \\
& Neg & 895.54 & 806.46 & 996.58 & \\
Duration (s) & Pos & 0.17 & 0.14 & 0.21 & $* *$ \\
& Neg & 0.42 & 0.34 & 0.51 & \\
AM extent (dB) & Pos & 5.77 & 4.60 & 7.30 & NS \\
& Neg & 5.67 & 4.50 & 7.24 & \\
Wiener entropy & Pos & -1.63 & -1.84 & -1.44 & $*$ \\
& Neg & -1.52 & -1.71 & -1.33 & \\
DFmin (Hz) & Pos & 846.64 & 778.01 & 921.82 & $*$ \\
& Neg & 964.72 & 899.40 & 1035.78 & \\
4th LPC coefficient (Hz) & Pos & 3913.22 & 3742.58 & 4069.90 & $*$ \\
& Neg & 4185.93 & 4020.41 & 4334.90 & \\
& & & & &
\end{tabular}


Some of these changes are in line with previous findings (e.g. spectral center of gravity, Leliveld et al. 2016; duration, Briefer et al. 2019). In particular, shorter durations in positive contexts have been observed across multiple species and could be a feature conserved throughout evolution (Briefer 2012). Overall, our results suggest that some parameters change with the valence experienced by pigs in a similar way across call types. These vocal parameters could be very useful for developing automated methods to monitor pig welfare on-farm.

\section{Acknowledgements}

This research is funded by the ERA-Net ANIHWA project SOUNDWEL.

\section{References}

Briefer, E. F. (2012). Vocal expression of emotions in mammals: mechanisms of production and evidence. Journal of Zoology, 288:1-20

Briefer, E. F., Vizier, E., Gygax, L., Hillmann, E. (2019). Expression of emotional valence in pig closedmouth grunts: Involvement of both source- and filter-related parameters. Journal of the Acoustical Society of America, In Press

Illmann, G., Hammerschmidt, K., Špinka, M. and Tallet, C. (2013). Calling by domestic piglets during simulated crushing and isolation: a signal of need? PLOS ONE, 8:e83529.

Leliveld, L. M. C., Düpjan, S., Tuchscherer, A., Puppe, B. (2016). Behavioural and physiological measures indicate subtle variations in the emotional valence of young pigs. Physiology \& Behavior, 157:116-124.

Linhart, P., Ratcliffe, V. F., Reby, D., Špinka, M. (2015). Expression of emotional arousal in two different piglet call types. PLoS ONE, 10:e0135414.

Mendl, M., Burman, O. H. P., Paul, E. S. (2010). An integrative and functional framework for the study of animal emotion and mood. Proceedings of the Royal Society B, 277:2895-2904.

Tallet, C., Linhart, P., Policht, R., et al (2013). Encoding of situations in the vocal repertoire of piglets (Sus scrofa): A comparison of discrete and graded classifications. PLoS ONE, 8:e71841. 